\title{
ANÁLISIS NEOTECTÓNICO EN EL PIEDEMONTE ORIENTAL DE CORDILLERA FRONTAL, ANDES CENTRALES. SAN JUAN, ARGENTINA
}

\author{
NEOTECTONIC ANALYSIS IN THE EASTERN PIEDMONT OF FRONTAL CORDILLERA, CENTRAL ANDES. \\ SAN JUAN, ARGENTINA
}

\author{
Juan Manuel ALCACER SANCHEZ, Laura Patricia A. PERUCCA \\ Universidad Nacional de San Juan, Facultad de Ciencias Exactas Fisicas y Naturales, Centro de Investigaciones de la Geosfera y \\ Biosfera. San Juan, Argentina. \\ Emails: alcacersanchez@hotmail.com; lperucca@unsj-cuim.edu.ar
}

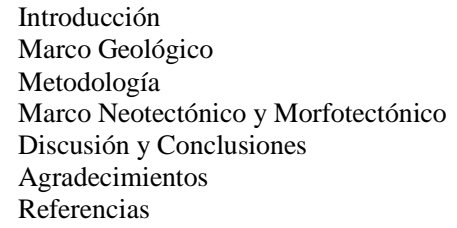

RESUMEN - El presente trabajo tiene por objetivo dar a conocer evidencias de actividad neotectónica sobre el Piedemonte Oriental de Cordillera Frontal, Andes Centrales, Argentina. La geomorfología de la región es compleja, producto de la interacción entre procesos sedimentarios, climáticos y tectónicos, predominando en los piedemontes de los principales cordones montañosos, sistemas de abanicos aluviales de edad pleistocena. La morfología de los sistemas aluviales ha sido modificada por la actividad tectónica vinculada al Sistema de Fallas Colangüil (SFC), el cual generó durante el Cuaternario, anomalías en la red de drenaje y un conjunto de escarpas a favor y en contra de la pendiente que afectan distintos niveles aluviales. Se diferenciaron 4 niveles aluviales; siendo $\mathrm{Q}_{1}$ el más reciente y coincidente con los actuales cauces y $\mathrm{Q}_{4}$ el más antiguo, el más elevado topográficamente y el menos preservado. En el tramo sur de este sistema se identificaron dos exposiciones naturales, denominadas Trinchera I y Trinchera II que permitieron conocer la cinemática de las fallas y los depósitos afectados. El análisis paleosismológico de las mismas indicaría la ocurrencia en el sector de al menos dos eventos sísmicos, el último de ellos con una edad inferior a 37.000 años. Finalmente no se descarta un tercer evento durante el Holoceno.

Palabras claves: Neotectónica, Morfotectónica, Sistema de Fallas Colangüil, Piedemonte.

\begin{abstract}
This paper aims to present evidence of neotectonic activity on the eastern piedmont of Cordillera Frontal, Central Andes, Argentina. The geomorphology of the region is complex, resulting of the interaction between sedimentary, climatic and tectonic processes, prevailing in the piedmonts of the main mountain ranges, alluvial fans systems which were deposited during the Pleistocene. The morphology of alluvial systems have been modified by the tectonic activity related to the Colangüil Fault System (CFS) that resulted in anomalies in the drainage network and a set of Quaternary favor-counter slope scarps affecting several alluvial levels. Thus, four alluvial levels were differentiated; being $\mathrm{Q}_{1}$ the most recent and coincident with existing drainage channels and $\mathrm{Q}_{4}$ the oldest, topographically highest and less preserved. Two natural exposures, called in this work Trench I and Trench II, which allowed knowing the kinematics of the faults and the affected deposits, were identified in the southern section of the Colangüil Fault System (SFC). The paleoseismological analysis suggests the occurrence of at least two seismic events in the area, implying that the last event have less than $~ 37,000$ years. Finally, the occurrence of a third event during the Holocene may be not ruled out.
\end{abstract}

Keywords: Neotectonics, Morphotectonics, Colangüil Fault System, Piedmont.

\section{INTRODUCCIÓN}

El área de estudio se encuentra en la región noroccidental de la provincia de San Juan, entre los $30^{\circ} 10^{\prime}$ y $30^{\circ} 20^{\prime} S$ y $69^{\circ} 10^{\prime}$ y $69^{\circ} 30^{\prime}$ O. Desde el punto de vista geológico el área de interés se corresponde con la porción septentrional de la depresión tectónica Uspallata - Calingasta Iglesia, estando la misma limitada al este por los cordones montañosos de Precordillera Occidental y por el oeste por la unidad montañosa de Cordillera Frontal (Figuras 1a, b, c).

Entre los paralelos $28^{\circ}$ y $33^{\circ} \mathrm{S}$, la placa de Nazca se introduce por debajo de la Sudamericana con actitud subhorizontal a unos $100 \mathrm{~km}$ de profundidad, a una tasa de 6,3 cm/año (Pardo Casas y Molnar 1987, Somoza 1998, Kendrick et al. 2003 - Figura 1b). Esta subhorizontalización se inició entre los 8 y 10
Ma (Jordan y Gardeweg 1987, Kay et al. 1991). La geometría sub-horizontal de la placa de Nazca a esta latitud es atribuida a la subducción de la dorsal Juan Fernández por debajo de la placa de América del Sur (Pilger, 1981; Anderson et al., 2007). En esta región se concentra gran parte de las deformaciones cuaternarias identificadas en la Argentina (Costa et al. 2000, Perucca y Vargas 2014).

En el piedemonte oriental de Cordillera Frontal, en el valle de Iglesia, se ubican tres sistemas de fallas paralelos de rumbo NNE, que afectan depósitos aluviales cuaternarios (Perucca y Martos 2009, 2012; Perucca y Vargas 2014). Estos sistemas fueron denominados por Perucca y Martos (2009, 2012) y Perucca y Vargas (2014) como Colangüil - Guañizuil, Pismanta - Las 


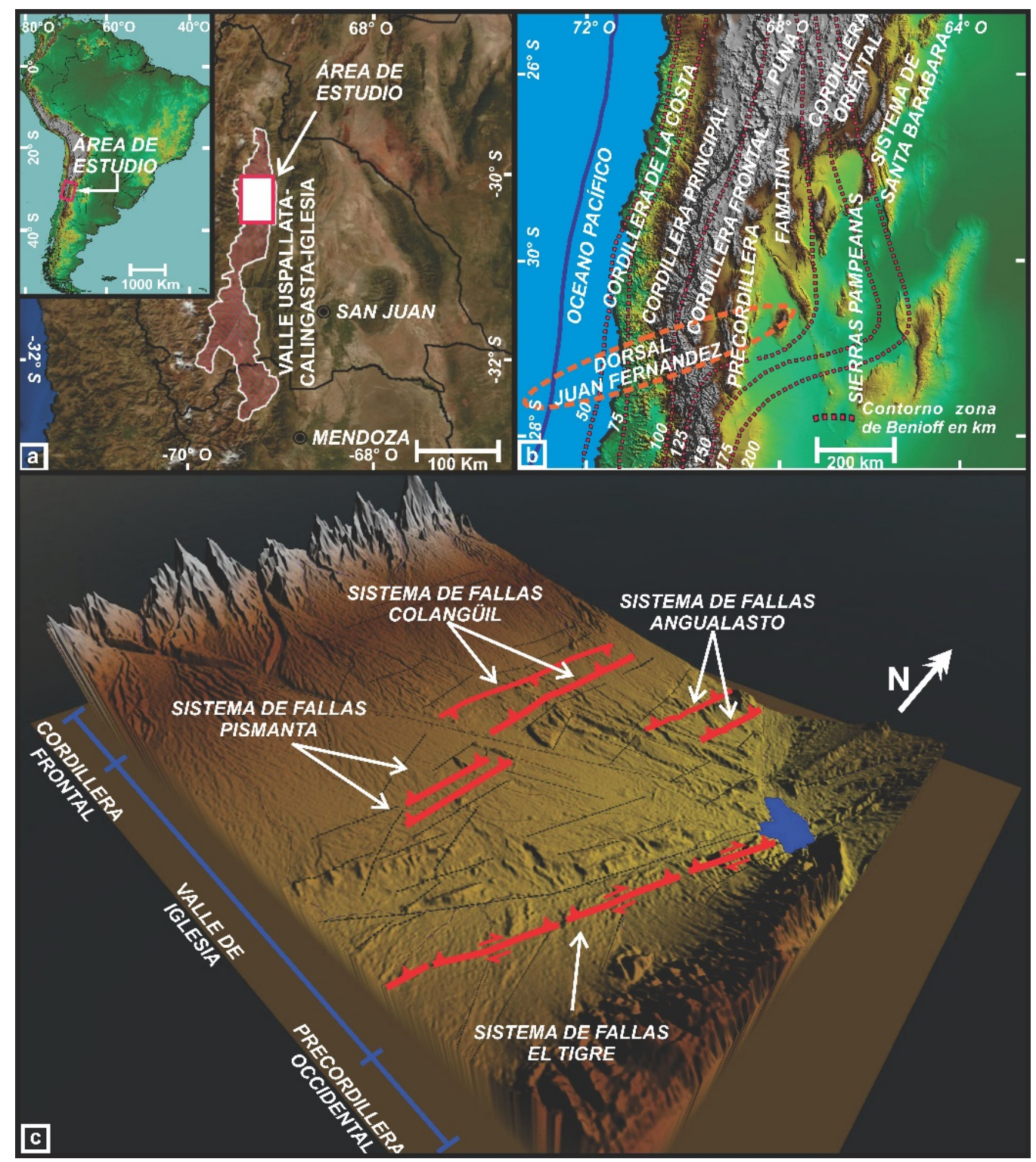

Figura 1 - a) Mapa de ubicación del área en el contexto sudamericano y regional correspondiente a la porción septentrional del Valle de Uspallata - Calingasta - Iglesia, San Juan, Argentina.; b) mapa de ubicación de la subducción pampeana (modificado de Anderson et al. 2007), indicándose la localización de la dorsal asísmica de Juan Fernández, de acuerdo a Yáñez et al. (2001) y las provincias geológicas reconocidas en el área de estudio; c) bloque 3D del valle de Iglesia, en el que se detallan las distintas unidades morfotectónicas y las principales estructuras con actividad tectónica pleistocena - holocena.

Flores y Bella Vista - Angualasto (Figura 1c). En general son fallas inversas, que inclinan en algunas secciones al este y en otras al oeste, casi siempre con ángulos elevados. Por otro lado, se puede mencionar el Sistema de Fallas El Tigre ubicado en el piedemonte occidental de Precordillera Occidental, definido por Bastías (1985) y descripto por Siame et al. (1997, 2006), Costa (2000) y Fazzito et al. (2013), entre otros autores.

De acuerdo con Perucca y Martos (2009, 2012), la geomorfología de la región es el resultado de la interacción entre los procesos sedimentarios, climáticos y tectónicos, siendo las geoformas predominantes los sistemas de abanicos aluviales originados durante el Pleistoceno por combinación de procesos aluviales y fluviales bajo condiciones climáticas pasadas más frías y húmedas, diferentes a las condiciones de aridez reinantes actualmente en la región.

Además, la morfología original del piedemonte oriental de los Andes Centrales a esta latitud ha sido modificada por la tectónica cuaternaria generando un conjunto de escarpas a contra pendiente, y alterando por consiguiente el patrón de drenaje en los distintos sectores a lo largo de la traza de los sistemas de fallas identificados.

La Falla Colangüil - Guañizuil, denominada 
en este trabajo Sistema de Fallas Colangüil (SFC) es una de las principales estructuras activas reconocidas en el piedemonte oriental de Cordillera Frontal. Se trata de un conjunto de secciones de falla con rumbo general nortesur, que afecta a los niveles aluviales del piedemonte y modifica la red de drenaje actual (Figuras 1c, 2a, b).
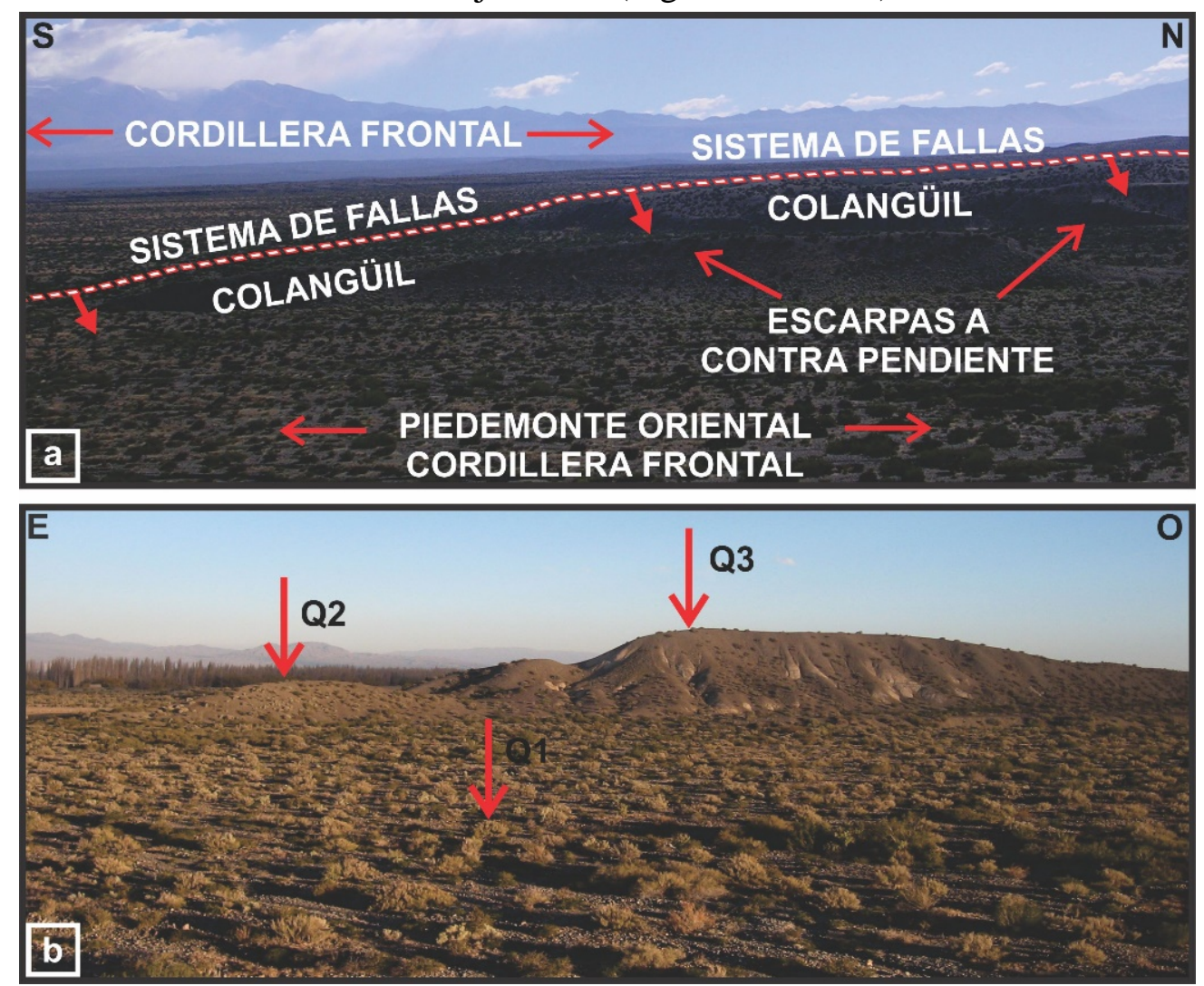

Figura 2 - a) Vista del Sistema de Fallas Colangüil sobre el piedemonte oriental de Cordillera Frontal, donde se aprecia la deformación tectónica de los diferentes niveles aluviales reconocidos en el área de estudio, mediante sistema de escarpas a contra pendiente, b) Niveles cuaternarios asociados al Sistema de Fallas Colangüil. Se denomina $\mathrm{Q}_{3}$ al nivel aluvial más antiguo reconocido en este sector de estudio y $\mathrm{Q}_{1}$ al nivel moderno coincidente con los actuales canales de drenaje.

En este trabajo se efectúa un análisis morfotectónico y paleosismológico preliminar del piedemonte oriental de los Andes Centrales en el valle de Iglesia, con el objetivo de ampliar el tiempo de observación y el nivel de actividad sísmica de esta estructura geológica. Además se tiene por primera vez un conocimiento básico de la geometría y la cinemática del Sistema de Fallas Colangüil, fundamental para estudios futuros de este sistema como fuente sismogénica potencial para la región.

\section{MARCO GEOLÓGICO}

En los cordones montañosos de Cordillera Frontal, al oeste del área analizada, afloran grauvacas, limoarcilitas y psamitas del Carbonífero Superior - Pérmico Inferior, granitos y granodioritas pérmicas, granitos del Mesozoico, sedimentitas continentales del Neógeno y depósitos glaciales, periglaciales, gravitacionales y fluviales del Pleistoceno Holoceno. Por su parte, en el ámbito de Precordillera Occidental, las unidades estratigráficas más antiguas corresponden a sedimentitas ordovícicas y devónicas cubiertas por depósitos neopaleozoicos, en los que se alojan cuerpos ácidos hipoabisales del Pérmico Inferior y el Triásico Inferior. El Paleógeno - Neógeno está representado por los depósitos continentales, tales como tobas, areniscas, pelitas y conglomerados y durante el Cuaternario se depositaron principalmente sedimentos continentales fluviales, gravitacionales y en menor proporción, eólicos (Perucca y Martos 2009, 2012).

En la porción occidental del valle de Iglesia, los depósitos cuaternarios están constituidos principalmente por clastos de 
grauvacas, cuarcitas y granodioritas. Los sedimentos más recientes ocupan la posición topográfica más baja, formando secuencias de tipo aluvial y fluvial cuya composición litológica está integrada por clastos de areniscas, grauvacas, lutitas y variedad de rocas ígneas. Los depósitos finos tales como limos, arcillas y arenas finas se ubican en la parte más baja de la depresión tectónica del Valle de Iglesia y constituyen los niveles de base locales de los cursos fluviales que atraviesan el valle.

Cristallini y Ramos (2000) señalaron que la evolución estructural de la región ubicada entre los paralelos $28^{\circ}$ y $33^{\circ} \mathrm{S}$ de los Andes es el resultado de la convergencia entre las placas de Nazca y América del Sur. Esta convergencia dio origen a un estilo de deformación de piel gruesa (thick skinned belt) en la Cordillera Frontal y una faja plegada y corrida con deformación de piel delgada (thin skinned fold and thrust belt) en la Precordillera.

Siame et al. (1996) consideraron que tanto el valle de Uspallata - Calingasta - Iglesia como la Precordillera corresponderían a una zona transpresiva de escala cortical entre los paralelos 30 y $31^{\circ} \mathrm{S}$, cuya deformación se distribuye con un movimiento horizontal lateral derecho a lo largo de la zona de fallamiento El Tigre y un movimiento inverso en la faja plegada y corrida de la Precordillera. De acuerdo a estos autores, el valle de Iglesia estuvo sometido a dos regímenes tectónicos contrastantes, uno compresivo durante el Paleozoico, con grandes acortamientos, y otro transpresivo a partir del Neógeno que modificó las estructuras pre-existentes.

Para Álvarez-Marrón (2006), los efectos combinados de compresión inicial y posterior transpresión han causado dificultades en la interpretación de las estructuras, sugiriendo que la deformación neógena no se adaptaría a un sistema de fajas plegadas y corridas, sino más bien a una geometría en flor positiva.

Por otra parte, Perucca y Bastías (2011) estimaron que en la Precordillera noroccidental de San Juan y La Rioja la deformación neotectónica posee una significativa componente transpresiva, con fallas inversas y pliegues, y con un desplazamiento lateral derecho $\mathrm{y}$ rumbo predominantemente submeridianal.

\section{METODOLOGÍA}

Los métodos aplicados en el análisis de la zona de estudio se basaron en la identificación de las distintas secciones del SFC con evidencia de actividad tectónica cuaternaria y en el análisis de la red de drenaje y reconocimiento de sus anomalías, a partir del análisis de imágenes satelitales y fotografías aéreas, definiendo de este modo aquellos sitios favorables para las tareas de campo.

Éstas consistieron en un estudio detallado de los indicadores morfotectónicos planares y lineales tales como determinación de los diferentes niveles aluviales, pendientes anómalas, escarpas a favor y en contra de la pendiente, cauces desplazados, obturados, colgados y con diferentes grados de incisión y vegetación alineadas, entre otras.

Además se efectuó una descripción somera de los depósitos cuaternarios afectados, la recolección de datos estructurales y la descripción de dos exposiciones naturales que permitieran determinar la cinemática de las fallas y los niveles cuaternarios involucrados.

\section{MARCO NEOTECTÓNICO Y MORFOTECTÓNICO}

Las mejores evidencias de actividad neotectónica en la región de interés se concentran en la porción media a distal del piedemonte oriental de Cordillera Frontal donde se destacan los sistemas de fallas Colangüil, Pismanta - Las Flores y Bella Vista - Angualasto (Perucca y Martos 2009, 2012, Perucca y Vargas 2014).

Se trata de un conjunto de niveles aluviales cuyas edades oscilan entre $\sim 180.000$ años y actuales (Tabla 1), ubicados a distintas alturas $\mathrm{y}$ afectados por fallas inversas, con escarpas en general a contra pendiente, y resaltos topográficos asimétricos con la cara libre de mayor pendiente al oeste de una altura variable que decrece de norte a sur (Figuras 1c, 3a).

Otra evidencia indirecta de actividad 
neotectónica son las anomalías observadas en la red de drenaje que es controlada por la presencia de escarpas a contrapendiente que

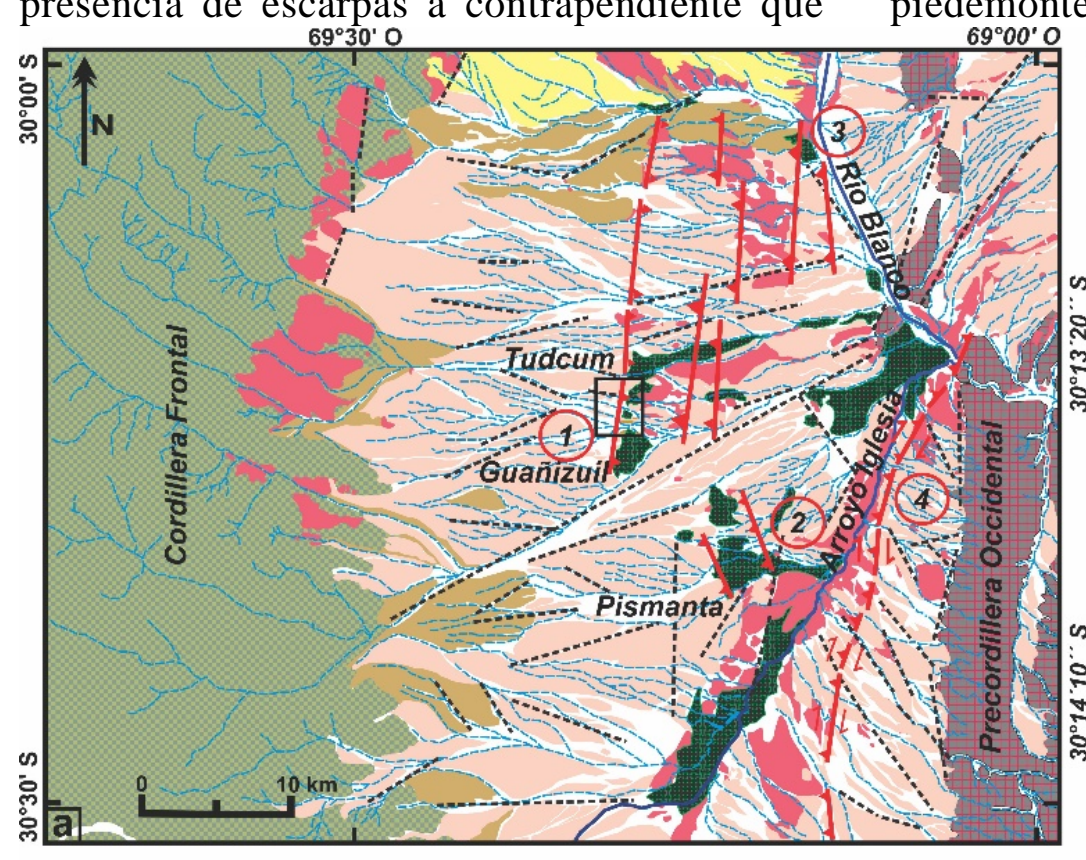

obturaron y/o desviaron los cauces de los arroyos temporales que atraviesan el piedemonte.

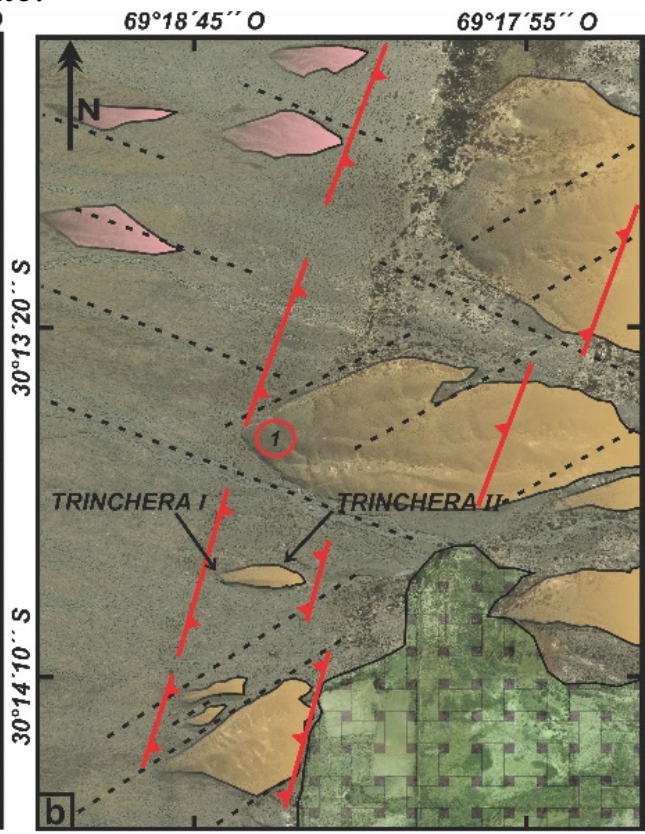

\section{REFERENCIAS}
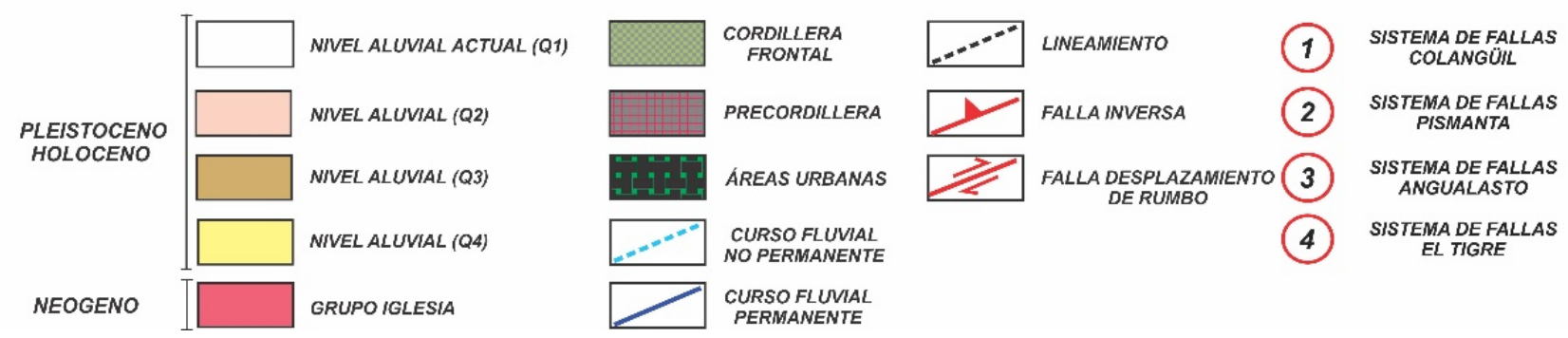

Figura 3 - a) Mapa geomorfológico regional en el que se indican los principales sistemas de fallas que afectan a la porción septentrional del valle de Uspallata - Calingasta - Iglesia, el conjunto de lineamientos secundarios y los diferentes niveles aluviales reconocidos en la región bajo estudio, b) Imagen de Google Earth de detalle del sector analizado, se observan dos secciones paralelas pero con distinta vergencia pertenecientes al Sistema de Fallas Colangüil, con la ubicación de las trincheras I y II.

Por otro lado, en el piedemonte occidental de Precordillera Occidental, se ubica el Sistema de Fallas El Tigre, con rumbo submeridianal y escarpas con la cara libre al este, a contrapendiente y con componente de rumbo dextral, definido por Bastías (1986) (Figura 3a).

Siame et al. (1997) señalaron que las fallas de este sistema afectan seis niveles de abanicos aluviales cuyas edades varían entre 700 ka para los más antiguos y 21 ka para las superficies más recientes hasta el nivel actual.

Fazzito (2011) señaló que se trata de una falla de alto ángulo cuyas irregularidades alternan tramos transtensivos y transpresivos, bajo un régimen regional transpresivo durante el Cuaternario. Pérez y Costa (2006) indicaron que las escarpas de las distintas secciones de este sistema aparecen discontinuas, con un patrón general en "V".

Asociados al conjunto de escarpas de falla expuestas en el piedemonte oriental de Cordillera Frontal y en el sector bajo estudio, se reconocieron 4 niveles aluviales cuaternarios, denominándose nivel $\mathrm{Q}_{1}$ al más reciente, coincidente con los actuales cauces de drenaje y $\mathrm{Q}_{4}$ al más antiguo, siendo este último el más elevado topográficamente y el de menor distribución areal (Figura 3a).

Las edades asignadas en forma preliminar a los distintos niveles cuaternarios (Tabla 1), se obtuvieron por correlación geomorfológica con los niveles aluviales datados por Siame et al. (1997) en el piedemonte occidental de la Precordillera, alrededor de $50 \mathrm{~km}$ al suroeste 
del sector analizado en este trabajo, y por las (2009, 2012) en el piedemonte oriental en el edades estimadas por Perucca y Martos valle de Iglesia.

Tabla 1 - Relación entre los distintos niveles cuaternarios observados en el área de estudio y sus correspondientes edades obtenidas mediante métodos radiométricos, modificado de Perucca y Martos (2009).

\begin{tabular}{|c|c|}
\hline NIVEL CUATERNARIO & EDAD ABSOLUTA (Años) \\
\hline NIVEL Q1 & $\mathbf{1 4 0 0} \pm \mathbf{1 3 0}$ \\
\hline NIVEL Q2 & $37000 \pm \mathbf{8 0 0 0}$ \\
\hline LACUSTRES & $>37000<\mathbf{1 0 0 0 0 0}$ \\
\hline NIVEL Q3 & $\mathbf{1 0 0 0 0 0} \pm \mathbf{2 1 0 0 0}$ \\
\hline NIVEL Q4 & $\mathbf{1 8 0 0 0 0} \pm \mathbf{3 8 0 0 0}$ \\
\hline${\text { NIVEL } \mathbf{Q 5}^{2}}^{2}$ & $\mathbf{3 8 0 0 0 0} \pm \mathbf{7 8 0 0 0}$ \\
\hline
\end{tabular}

${ }^{1}$ nivel aluvial más antiguo definido para el área de estudio en el presente trabajo.

${ }^{2}$ nivel aluvial reconocido en el piedemonte de la sierra del Tigre por Siame et al (1997).

En el sitio de estudio y a partir de las observaciones de campo, fue posible diferenciar 3 niveles aluviales, siendo Q3 el más antiguo en este sector y Q1 el actual, reconociéndose además depósitos caracterizados por el desarrollo de facies lacustres, los cuales posiblemente estarían vinculados a las zonas de escarpas de falla que endicaron los pequeños arroyos provenientes del sector montañoso. Estos depósitos finos han sido cronoestratigráficamente ubicados en el presente trabajo entre el Nivel $\mathrm{Q}_{2} \mathrm{y}$ el nivel aluvial anterior o Nivel $Q_{3}$ (Figura 3b).

Se les asigna tentativamente una edad pleistocena tardía - holocena ( $<100 \mathrm{ka})$, interpretándose como pequeñas lagunas someras y temporarias (del tipo sagponds) originadas al pie de la escarpa de las secciones de falla del SFC.

Asociado al Sistema de Fallas Colanguil, de rumbo promedio $\mathrm{N}^{\circ} \mathrm{E}$ a $\mathrm{N} 15^{\circ} \mathrm{E}$, se reconoció además un conjunto de lineamientos secundarios con dos orientaciones predominantes: NE y NO (Figura 3b).

Por otro lado, la existencia de anomalías en la red de drenaje proporciona indicios indirectos sobre la existencia de fallas activas en una determinada región. En el piedemonte oriental de la Cordillera Frontal se desarrolla una red de drenaje dendrítica divergente, la cual sería resultado del pasaje de los cauces fluviales de la zona montañosa al piedemonte controlado principalmente por la pendiente del terreno; sin embargo, hacia las proximidades al SFC el diseño divergente es reemplazado localmente por un diseño en rastrillo, característico de zonas tectónicamente activas debido a que los cursos de agua se agrupan aumentando su caudal a fin de poder vencer la estructura activa (e.g. Audemard, 1999).

En este caso, las escarpas de falla oponen resistencia a la normal escorrentía sobre piedemonte cordillerano ocasionando la conjunción de varios canales de drenaje, a fin de poder superar dichas escarpas.

En la figuras $4 \mathrm{a}$ y $4 \mathrm{~b}$ se detalla, de norte a sur, el conjunto de anomalías observadas en la red de drenaje y la vegetación alineada según el trazo de las distintas secciones de falla.

En la imagen es posible diferenciar aquellos sectores donde la red adquiere un diseño en rastrillo en relación con la traza de falla. El análisis de la imagen, sumado a las observaciones de campo también permitió la diferenciación entre el conjunto de canales abandonados, que no pudieron superar la barrera topográfica generada por la escarpa de falla, de aquellos canales actualmente activos por donde hoy en día discurren las aguas. 

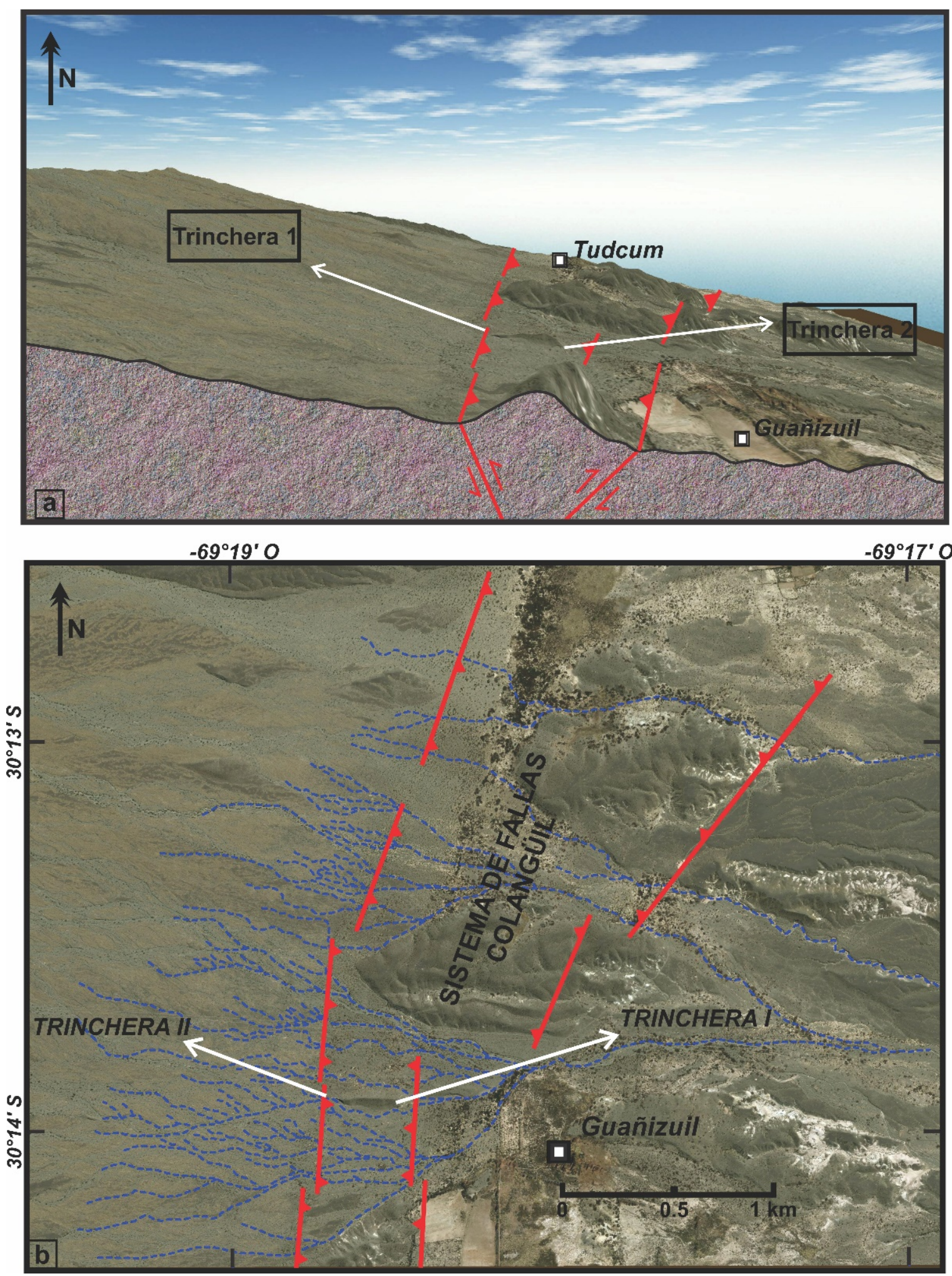

Figura 4 - a) Bloque 3d representativo de la pendiente topográfica, distribución de canales de drenaje y escarpas a contra pendiente asociadas a la deformación tectónica ocasionada por el Sistema de Fallas Colangüil, b) Imagen de Google Earth con las anomalías de la red de drenaje observadas en el área de estudio, en ella puede observarse el diseño en rastrillo que adquieren los canales al alcanzar la escarpa de falla.

En una lomada piedemontana correspondiente al nivel aluvial $\mathrm{Q}_{3}$ y de orientación oeste-este, se identificaron dos exposiciones naturales, denominadas en este trabajo trincheras I y II, sobre las cuales fue posible reconocer depósitos aluviales cuaternarios afectados por fallas inversas y un conjunto de evidencias paleosísmicas tales como cuñas coluviales, diques de arenas, brechas de falla y clastos con el eje mayor paralelo al plano de falla (Figuras $5 \mathrm{y}$ 6). 


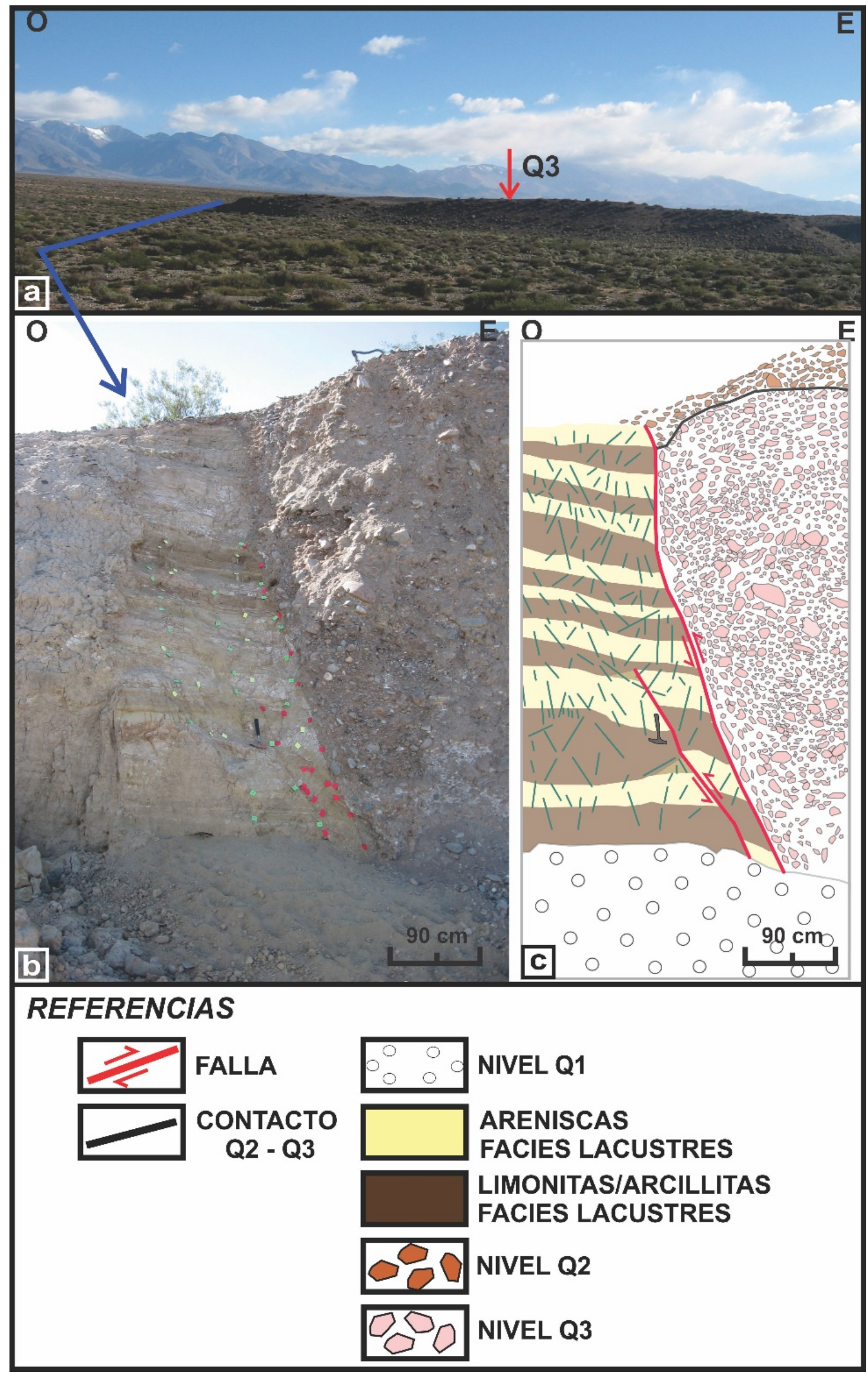

Figura 5 - a) Fotografía de la lomada pedemontana correspondiente al nivel aluvial $\mathrm{Q}_{3}$, donde se exponen las trincheras naturales I y II, b) Fotografía de la exposición natural ubicada en el extremo occidental de la lomada localizada en la inmediaciones de Guañizuil, denominada Trinchera I, c) Interpretación de la Trinchera I, se observa el contacto por falla entre los depósitos aluviales correspondientes al nivel aluvial $\mathrm{Q}_{3}$ que sobreyacen facies lacustres. En la imagen se observa que el nivel aluvial $\mathrm{Q}_{2}$ también se encuentra afectado por la falla. 


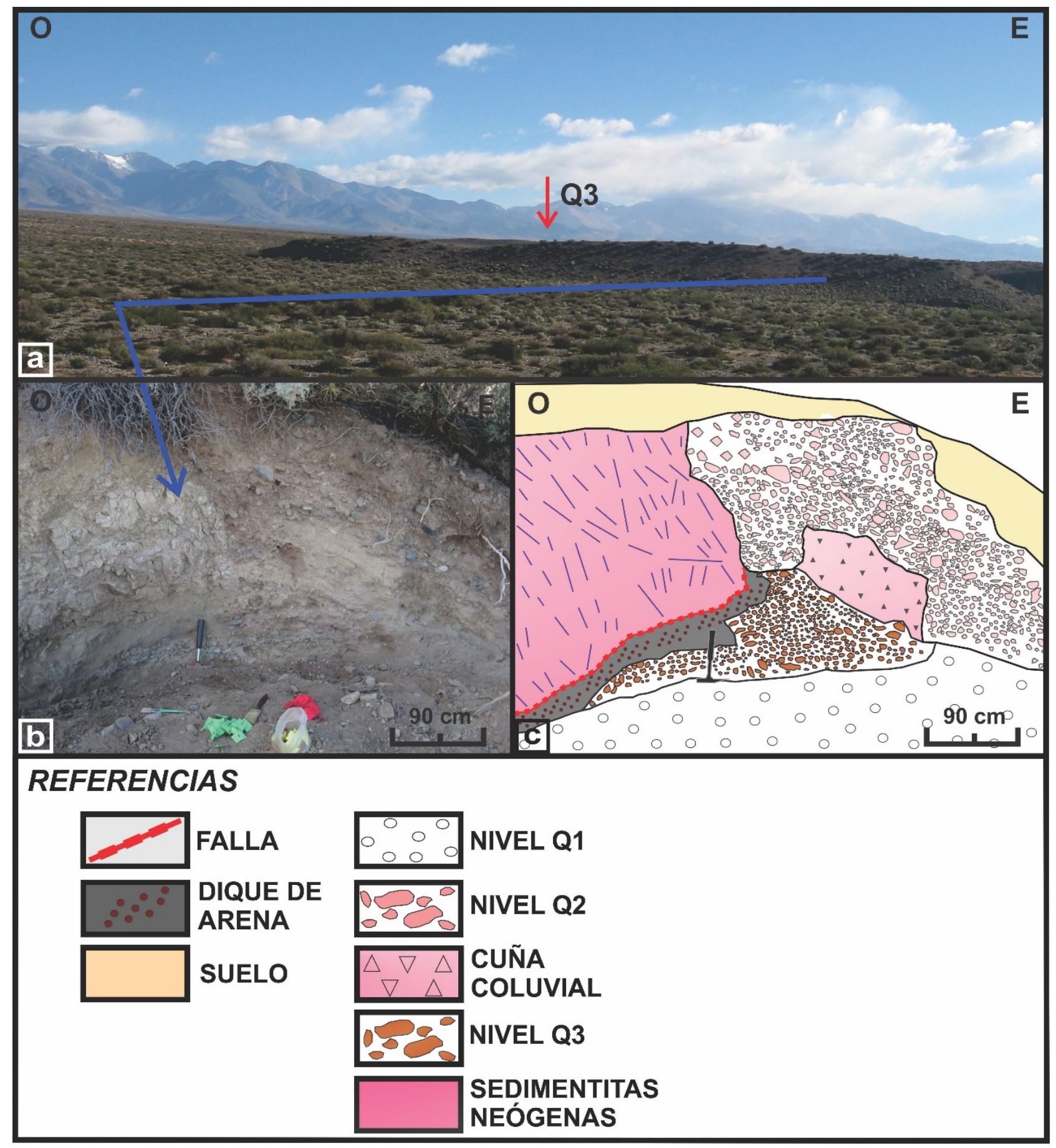

Figura 6. a) Fotografía de la lomada pedemontana correspondiente al nivel aluvial $\mathrm{Q}_{3}$, donde se exponen las trincheras de falla I y II, b) Fotografía de la exposición natural ubicada en el extremo oriental de la lomada localizada en la inmediaciones de Guañizuil, denominada Trinchera II, c) Interpretación de la Trinchera II, en la que se observa el contacto tectónico entre las sedimentitas neógenas y los depósitos aluviales asignados al Nivel Q3; además un dique de arena en el plano de falla y una cuña coluvial constituida por clastos de sedimentitas neógenas. Por encima se ubica un nivel edáfico muy incipiente que se encuentra suavemente flexurado.

En la Trinchera I, ubicada en el extremo oeste de la lomada (Figura 5a) se observa una falla que pone en contacto depósitos de facies gruesas aluviales correspondientes al nivel $\mathrm{Q}_{3}$, de edad pleistocena media a tardía, sobre los depósitos de las facies lacustres.

La falla es inversa con rumbo $\mathrm{N} 20^{\circ} \mathrm{E}$, inclina al $\mathrm{E}$ entre $54^{\circ}$ en la parte inferior y $82^{\circ}$ en la parte superior del plano de falla. En este sector también es posible observar que los clastos de los depósitos de las facies gruesas atribuidos al Nivel $\mathrm{Q}_{3}$ situados próximos al plano de falla han sido rotados disponiéndose con sus ejes mayores de forma paralela a dicho plano lo que implicaría que el movimiento de la falla ha sido fundamentalmente paralelo al buzamiento del 
plano, si bien no se descarta una componente horizontal secundaria (Figuras 5 b, c).

Se observa además hacia el tope de la exposición estudiada que los depósitos de facies aluviales gruesas atribuidas al Nivel $Q_{2}$ también han sido afectados por la falla. La altura de la escarpa, que mira al oeste, es de aproximadamente $2,50 \mathrm{~m}$.

Sobre la base del análisis de la Trinchera I se sugiere la ocurrencia de al menos tres eventos sísmicos durante el Pleistoceno medio a tardío, uno que originó la escarpa que provocó la formación del cuerpo lacustre y afectó el nivel $\mathrm{Q}_{3}(<100 \mathrm{ka})$, otro que colocó estos depósitos aluviales por encima del cuerpo lacustre y el último evento que afectó al nivel $\mathrm{Q}_{2}(<37 \mathrm{ka})$.

En la Trinchera II se observa una falla inversa de rumbo $\mathrm{N} 12^{\circ} \mathrm{E}$ que inclina $35^{\circ} \mathrm{E}$ y pone en contacto sedimentitas neógenas con depósitos de facies gruesas aluviales correspondiente al nivel $\mathrm{Q}_{3}$. Además se reconoció una cuña coluvial, con fragmentos de rocas de edad neógena (Figuras 6 b y 6 c). No se han identificado estrías en el plano de falla aunque se estima, debido a su bajo ángulo de buzamiento, un corrimiento (thrust) con movimiento principal vertical.
Hacia la parte superior de la trinchera se ubican incipientes horizontes edáficos fundamentalmente de tipo esquelético, lo cual sugeriría una estabilidad tectónica posterior al evento sísmico para dicho sector que permitió la generación del suelo.

También fue posible reconocer paralelo al plano de falla un dique de arena de 40 a $60 \mathrm{~cm}$ de espesor, cuyo origen ha sido interpretado en este trabajo como asociado a la ocurrencia de procesos de licuefacción durante eventos sísmicos prehistóricos (Figuras 6 b y 6 c). Estos diques de arena ya han sido descriptos en exposiciones analizadas en otras estructuras activas de la región por Perucca et al. (2009) y Perucca y Vargas (2014) y también han sido registrados durante los sismos históricos ocurridos en la provincia de San Juan (Perucca et al. 2006).

El análisis de las evidencias observadas en la Trinchera II sugiere la ocurrencia al menos un evento sísmico y al igual que en el caso anterior, la falla en este sector afecta al nivel $\mathrm{Q}_{3}$ $\mathrm{y}$ por consiguiente la edad del último evento reconocido sería $<100 \mathrm{ka}$. Sin embargo, el nivel ubicado por encima con incipiente edafización se encuentra flexurado, posiblemente indicando un evento sísmico posterior a su depositación.

\section{DISCUSIÓN Y CONCLUSIONES.}

El conjunto de evidencias tanto directas como indirectas observadas en el piedemonte oriental de Cordillera Frontal, en el valle de Iglesia, permitió evaluar en forma cualitativa y preliminar, la actividad tectónica cuaternaria del Sistema de Fallas Colangüil.

Se identificaron 4 niveles aluviales, designándose como $\mathrm{Q}_{4}$ al nivel más antiguo y $\mathrm{Q}_{1}$ al actual nivel de acumulación. Los distintos niveles aluviales reconocidos en el área fueron definidos sobre la base de su granulometría, posición topográfica y por la presencia e intensidad de pavimento y/o barniz del desierto. Si bien no se descarta el condicionante climático para esta región, la existencia de los 4 niveles aluviales estaría vinculada principalmente al Sistema de Fallas Colangüil y a la ocurrencia de posiblemente al menos 3 eventos sísmicos en los últimos 180.000 años. Dichos niveles aluviales se encuentran restringidos al área de fallamiento, desapareciendo hacia el sur y norte por debajo de depósitos aluviales provenientes de Cordillera Frontal. Perucca y Martos (2009) indicaron que la morfogénesis del abanico aluvial más antiguo, denominado en este trabajo nivel $\mathrm{Q}_{4}$ (Pleistoceno medio a tardío) habría finalizado por procesos tectónicos que favorecieron su incisión, quedando solamente relictos de este nivel en el área de estudio. Según estos autores, la influencia de los cambios climáticos con alternancia de climas más húmedos y fríos en las áreas montañosas generaron los niveles aluviales denominados en este trabajo como $\mathrm{Q}_{3}$ y $\mathrm{Q}_{2}$. A esto se sumó la actividad tectónica puesta de manifiesto por las fallas que afectan la región y deforman todos los niveles aluviales a excepción del actual.

El reconocimiento de marcadores lineales tales como las anomalías observadas en la red de drenaje y vegetación a lo largo del trazo de las distintas secciones de falla también constituye, aunque de forma indirecta, evidencia de la actividad tectónica cuaternaria 
del sistema de fallas. El desarrollo de un diseño de red de drenaje en rastrillo supone la migración de los canales de escurrimiento superficial a fin de superar las distintas escarpas de falla a contrapendiente que se localizan sobre el piedemonte cordillerano. Sobre la sección de falla ubicada en el extremo sur del sistema de fallas, se ubicaron dos exposiciones naturales denominadas, Trinchera I y II, en las que se infirieron al menos dos (y probablemente) tres eventos sísmicos.

La falla identificada en la Trinchera I ubicada al oeste es inversa, posee un rumbo submeridianal e inclina con alto ángulo al este colocando los depósitos fanglomerádicos del nivel $\mathrm{Q}_{3}$ por encima de sedimentos lacustres ubicados al pie de la escarpa a contrapendiente. Por encima se ubican depósitos del nivel $\mathrm{Q}_{2}$ también deformados.

En la trinchera II, la falla inversa inclina al oeste con bajo ángulo, afecta los depósitos del nivel $\mathrm{Q}_{3}$ y presenta una cuña coluvial constituida por fragmentos correspondientes a las sedimentitas neógenas y un dique de arena en el plano de falla posiblemente relacionado a un proceso de paleolicuefacción.

La edad del evento más reciente identificado para este sistema sería inferior a 37 ka, que es la edad asignada tentativamente al nivel aluvial $\mathrm{Q}_{2}$ sobre la base de su correlación con las edades absolutas obtenidas por Siame et al. (1997) para los niveles aluviales ubicados en el piedemonte occidental de la Precordillera Occidental (Perucca y Martos, 2009, 2012) con los niveles aluviales del piedemonte oriental de Cordillera Frontal. Sin embargo, en la trinchera II se identificó un nivel edáfico suavemente flexurado por la falla, por lo que se estima un evento sísmico posterior a su formación, ya en tiempos holocenos.

De acuerdo a Ré et al. (2003), la evolución de la cuenca de Iglesia estaría vinculada a un sistema de corrimientos (tipo piggyback) que habría comenzado aproximadamente a los 20 $\mathrm{Ma}$, continuando su actividad hasta la actualidad con una importante componente de acortamiento horizontal. En esta región andina, la subducción plana se atribuye a la subducción de la dorsal de Juan Fernández por debajo de la placa sudamericana (Pilger, 1981), que habría generado la faja plegada y corrida de piel fina con vergencia oriental en el valle de Iglesia.

Por otro lado y tal como lo señalaran Pérez y Costa (2006), las diferencias en el patrón de afloramiento de las fallas ubicadas en el valle de Iglesia podrían también indicar una distribución de movimiento característico de secciones terminales de varias fallas transcurrentes. Estos autores manifestaron que las distintas secciones de falla estarían controladas por diferentes anisotropías precuaternarias y por lo tanto tendrían distintos comportamientos cinemáticos.

Las exposiciones naturales descriptas en nuestro trabajo y el análisis geomorfológico efectuado, con escarpas que indican un desplazamiento principal vertical, arroyos controlados por las estructuras con formación de sagponds al pie de las escarpas, etc., permiten inferir para este sistema de fallas una componente vertical principal, en la que el bloque elevado se mueve relativamente sobre el bloque descendido. Sin embargo, no se debe descartar que las secciones de fallas analizadas puedan ser secciones que forman parte del Sistema de Fallas El Tigre, en un arreglo del tipo cola de caballo o en escalón hacia la izquierda, compatible con un régimen transpresivo para el sector.

Por todo lo expuesto, la potencialidad del SFC y de los sistemas de fallas ubicados en ambos piedemontes del valle de Iglesia, cercanos a numerosos poblados, proyectos mineros y a un embalse ubicado sobre el río Jáchal, no debe ser subestimada, siendo necesario incrementar los estudios de detalle en la región, profundizar en los estudios de los indicadores cinemáticos y paleosismológicos a través de la confección de trincheras a lo largo de las estructuras, caracterizar la falla en subsuperficie utilizando geofísica (gravimetría y sísmica, entre otros) y efectuar dataciones absolutas en los distintos niveles afectados por las fallas con el fin de determinar cuantitativamente el peligro sísmico de esta región.

\section{AGRADECIMIENTOS}

La presente contribución fue financiada con fondos del proyecto 1 E/850 CS-CICITCA, así como del PIP 0799/247 (CONICET). 


\section{REFERENCIAS}

ÁLVAREZ MARRÓN, J. Neogene structures overprinting Paleozoic thrust systems in the Andean Precordillera at $30^{\circ} \mathrm{S}$ latitude. Journal of the Geological Society, v. 163, p. 949964, 2006.

ANDERSON, M; ALVARADO, P; ZANDT, G; BECK, S. Geometry and brittle deformation of the subducting Nazca Plate, central Chile and Argentina: Geophysical Journal International, v. 171, n. 1, p. 419-434, 2007.

AUDEMARD, F. Morpho-structural expression of active thrust fault systems in the humid tropical foothills of Colombia and Venezuela. Zeitschrift für Geomorphologie, v. 118, p. 1-18, 1999.

BASTÍAS, H. Fallamiento cuaternario en la región sismotectónica de Precordillera. San Juan, 1986. 160p. Tesis (Doctorado en Ciencias Geológicas), Facultad de Ciencias Exactas, Físicas y Naturales, Universidad Nacional de San Juan.

COSTA, C; MACHETTE, M; DART, R; BASTÍAS, H; PAREDES, J; PERUCCA, L; TELLO, G; HALLER, K. Map and Database of Quaternary Faults and Folds in Argentina. U.S. Geological Survey Open-File Report 000108, 75p, 2000.

CRISTALLINI, E \& RAMOS, V. Thick-skinned and thinskinned thrusting in La Ramada fold and thrust belt: Crustal evolution of the High Andes of San Juan, Argentina ( $32^{\circ} \mathrm{SL}$ ). Tectonophysics, vol. 317, p. 205-235, 2000.

FAZZITO, S. Estudios geofísicos aplicados a la geotectónica de la falla El Tigre, Precordillera de San Juan. Buenos Aires, 2011. 227 p. Tesis (Doctorado en Ciencias Geológicas), Facultad de Ciencias Exactas, Físicas y Naturales, Universidad de Buenos Aires.

FAZZITO, S; CORTÉS, J; RAPALINI, A; TERRIZZANO, C. The geometry of the active strike-slip El Tigre Fault, Precordillera of San Juan, Central-Western Argentina: integrating resistivity surveys with structural and geomorphological data. International Journal of Earth Sciences, v. 102, p. 1447-1466, 2013.

JORDAN, T \& GARDEWEG, M. Tectonic Evolution of the Late Cenozoic Central Andes $\left(20^{\circ}-33^{\circ}\right.$ S). In: Mesozoic and Cenozoic Evolution of the pacific Ocean Margins Oxford Univ. Press. N.Y., edit. Z. Ben-Avraham, p. 193-207, 1987.

KAY, M; MPODOZIS, C; RAMOS, V; MUNIZAGA, F. Magma source variations for mid-late Tertiary magmatic rocks associated with shallowing zone and thickening crust in the central Andes $\left(28^{\circ}\right.$ to $\left.33^{\circ} \mathrm{S}\right)$. In: Harmon, R. S. and Rapela, C. W. (Eds.), Andean magmatism and its tectonic setting, Geological Society of America, Special Paper 265, p.113-137, 1991.

KENDRICK, E; BEVIS, M; SMALLEY, R; BROOKS, B; VARGAS, R; LAURÍA, E; FORTES, L. The Nazca-South America Euler Vector and its Rate of Change. Journal of South American Earth Sciences, vol. 16, p. 125-131, 2003.

PARDO CASAS, F \& MOLNAR, P. Relative motion of the Nazca (Farallón) and South America plate since late Cretaceous times. Tectonics, vol.6, p. 233-248, 1987.

PÉREZ, I \& COSTA, C. El sistema de fallamiento El Tigre entre el río Jáchal y el cerro Negro de Iglesia. XIII Reunión de Tectónica, San Luis, 2006. Resúmenes p. 48, 2006.

PERUCCA, L \& BASTIAS, H. Quaternary active transpression in the north of Western Precordillera (San Juan and La Rioja, Argentina). Cenozoic Geology of the Central Andes of Argentina. In: Salfity, J.A., and Marquillas, R.A., eds., Cenozoic geology of the Central Andes of Argentina. Instituto del Cenozoico, Universidad Nacional de Salta, Salta, p. 327-334, 2011.

PERUCCA, L \& MARTOS L. Análisis de la evolución del paisaje cuaternario del Valle de Iglesia, San Juan. Revista de la Asociación Geológica Argentina, v. 65, p. 624-637, 2009. PERUCCA, L \& MARTOS, L. Geomorphology, tectonism and Quaternary landscape evolution of the Central Andes of San Juan $\left(30^{\circ} \mathrm{S}-69^{\circ} \mathrm{W}\right)$, Argentina. Quaternary International, v. 253, p. 80-90, 2012.

PERUCCA, L \& VARGAS, N. Neotectónica de la provincia de San Juan, centro-oeste de Argentina. Boletín de la Sociedad Geológica Mexicana, v. 66, p. 291-304, 2014.

PERUCCA, L.; MOREIRAS, S.; BRACCO, A. Determination of Seismogenic Structures and Earthquake Magnitude from Seismites in Holocene Lacustrine Deposits. Precordillera Range, Central-Western Argentina. Journal of Iberian Geology, p. 10-20, 2009.

PILGER, R. Plate reconstructions, aseismic ridges and low angle subduction beneath the Andes. Geological Society of America Bulletin, v. 92, p. 448-456, 1981.

RE, G. H.; JORDAN, T. E.; KELLEY, S. Cronología y paleogeografía del Terciario de la Cuenca Intermontana de Iglesia septentrional, Andes de San Juan, Argentina. Revista de la Asociación Geológica Argentina, v. 5, n. 8, p. 31-48, 2003.

SIAME, L; SEBRIER, M; BELLIER, O; BOURLES, D; CASTANO, J; ARAUJO, M; YIOU, F; RAISBECK, G. Segmentation and horizontal slip-rate estimation of the El Tigre Fault Zone, San Juan Province (Argentina) from SPOT images analysis. Third ISAG (St Malo, France), Extended Abstracts...St Malo, p. 239-242, 1996.

SIAME, L; SÉBRIER, M; BELLIER, O;BOURLES, D; CASTANO, J; ARAUJO, M.Geometry, segmentation and displacement rates of the El Tigre fault, San Juan Province (Argentina) from SPOT image analysis and 10Be dating's. Annales Tectonicae, International Journal of Structural Geology and Tectonics, p. 1-2, 3-26, 1997.

SIAME, L; BELLIER, O; SEBRIER, M. Active tectonics in the Argentine Precordillera and western Sierras Pampeanas. Revista de la Asociación Geológica Argentina, v. 61, p. 604-619, 2006.

SOMOZA, R. Updated Nazca (Farallón). South America relative motions during the last $40 \mathrm{My}$ : implications for mountain building in the central Andean region. Journal of South American Earth Sciences, v. 11, p. 211-215, 1998.

YÁÑEZ, G.; RANERO, G.; VON HUENE, R.; DÍAZ, J. Magnetic anomaly interpretation across a segment of the Southern Central Andes (32-34 $\left.{ }^{\circ} \mathrm{S}\right)$ : implications on the role of the Juan Fernández Ridge in the tectonic evolution of the margin during upper Tertiary. Journal of Geophysical Research, v. 106, p. 6325-6345, 2001.

Submetido em 23 de fevereiro de 2017 Aceito em 14 de outubro de 2017 